\title{
TARGET ANXIETY
}

NEW YORK-Anxiety is a pressing problem. It affects about 10 percent of market for anxiolytics of roughly $\$ 1.2$ billion. Benzodiazepines-including Valium and Xanax-captured about 90 percent of this market, despite severe side effects like addiction and sedation.

Two biotechnology companies-

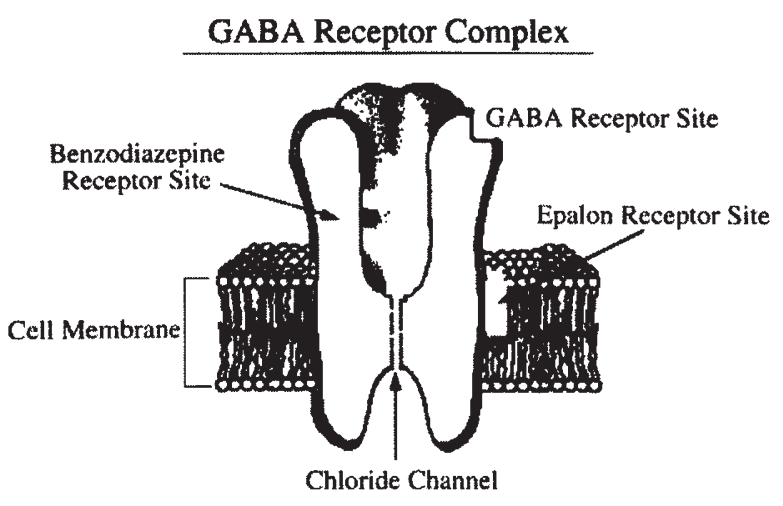
the U.S. population, creating a 1990

side effects. CoCensys and Neurogen, conversely, intend to develop compounds that bind specifically to individual receptor subtypes. Receptor-specific binding should overcome the side-effect problems of benzodiazepines, while maintaining benzodiazepine efficacy.

Both companies are taking drug-development approaches that combine conventional pharmaceutical chemistry with molecular biology. Conventional chemistry lets them synthesize drugs specific for receptor subtypes. Molecular biology lets them produce in mammalian cells proteins making up each receptor subtype. Such cells serve as screens for drugs binding specifically to single subtypes. Neurogen-a fouryear-old company that lost $\$ 3$ million in 1990-

Neurogen (Branford, CT) and CoCensys (Menlo Park, CA) - are trying to develop anxiolytics as effective as benzodiazepines but without their side effects. They hope the products impact the anxiolytic market the way Eli Lilly's (Indianapolis, IN) Prozac impacted antidepressant sales. Prozac's introduction caused the U.S. antidepressant market to more than double, from $\$ 400$ million in 1988 to $\$ 1$ billion in 1990 , primarily because it caused fewer side effects. "Prozac's success shows that modest improvements in side-effect profiles can dramatically capture and expand existing markets," says Mark Simon, a biotech analyst at Robertson Stephens (San Francisco, CA).

Neurogen and CoCensys are at similar stages of development. Both companies expect to enter products in clinical trials this year. And both anticipate alliances with major drug companies soon.

Both firms are targeting the gammaaminobutyric acid (GABA) receptor. GABA, the most common inhibitory neurotransmitter, calms excited neurons. When GABA binds to its receptors on the outer membranes of excited neurons, it briefly opens chloride channels in the membranes (diagram). Chloride ions flow into the neurons, calming the cells so they can fire again.

The GABA receptor is actually made up of several receptor subtypes. Each has its own function, researchers believe, with one playing a role in anxiety, another in sleep, and another in epileptic convulsions. Benzodiazepines, for their part, bind nonspecifically to the various receptor subtypes and, indeed, such binding is what causes their unwanted calls its lead compound NGD 91-1. The
compound, which binds to about 20 percent of GABA receptors in primate cortical tissue, has a molecular weight under 500. Lipophilic qualities allow it to pass through the blood-brain barrier.

Neurogen is also developing compounds that target sleep disorders and cognition. The company's hypnotics, like its anxiolytics, would bind to specific GABA receptors to increase GABA's calming effect. Its cognitive enhancers,

\section{TARGETING RIBOSOMAL RNA \\ IDENTIFYING MICROBES}

LONDON-"We believe that a rapid and sensitive method of directly identifying and enumerating microorganisms in natural samples is now within reach," says Rudolf Amann of Munich Technical University in Germany. He bases his claim on a two-part procedure which he and colleagues have developed to target ribosomal RNA (rRNA) in single bacterial cells. First, they apply an oligonucleotide, labelled with digoxigenin, which hybridizes with the relevant rRNA. The complex is then revealed by a second probe composed of anti-digoxigenin antibody fragments labelled with either alkaline phosphatase or horseradish peroxidase, whose activity is detected by conventional techniques. The amplification made possible by the enzyme should commend the method for use in monitoring engineered organisms after being released into the environment.

The Munich researchers have been trying to increase the sensitivity of methods of identifying single cells through their rRNA, for applications in micro- on the other hand, would decrease GABA's effect. Such a decrease could boost the action of neurotransmitters like acetylcholine and catecholamine that contribute to cognition. Sleep disorders affect 20 million people in the U.S., while 3.5 million Americans suffer cognitive impairment from such neurodegenerative diseases as Alzheimer's and Parkinson's.

CoCensys-a private company founded in 1989-is developing a family of compounds, called epalons, that form when the body breaks down the steroid hormone progesterone. CoCensys increases the compound's half life by "adding a couple more atoms" to block enzymatic degradation, says Robert McNeil, the company's chairman. He adds that the compounds have no affinity for steroid-hormone receptors.

CoCensys will initially target its compounds against types of epilepsy known as infantile spasms and petit-mal seizures. In all, about 1.6 million Americans suffer from epilepsy. CoCensys will also target anxiety and sleep disorders, as well as premenstrual syndrome (PMS). About 45 million women in the U.S. are afflicted with PMS, which is associated with a drop in progesterone levels before menstruation.

Whether CoCensys or Neurogen will develop compounds that compete with benzodiazepines is uncertain. "Clinical trials will provide the answer," says CoCensys' McNeil. -B.J. Spalding

bial ecology. They decided to experiment with enzymes, detectable through their catalytic activities, after failing to improve signal intensity significantly by directing more than one marker molecule to each target molecule. For example, oligonucleotide probes covalently bound to multiple flourescent dye molecules did not yield worthwhile levels of amplification.

A major question about linking antibody fragments with enzymes was whether such large complexes would pass through the bacterial cell wall. After initial lack of success, Amann and his co-workers have now found that the complexes can be made to penetrate, by treating the cells with lysozyme and EDTA. They have, for example, used a probe for the 23S rRNA of Pseudomonas cepacia to detect this organism specifically in a mixed culture. The only limitation of the new technique, which is probably related to the structure of the cell wall, is that it only works with Gram negative bacteria. -Bernard Dixon 